\title{
ANÁLISE QUANTITATIVA DA MELHORA NA FUNÇÃO PULMONAR E DA FORÇA MUSCULAR RESPIRATÓRIA ENTRE O PRÉ E PÓS-OPERATÓRIO DE CIRURGIA DE GASTRECTOMIA VERTICAL POR VIDEOLAPAROSCOPIA
}

\author{
Nelson Coimbra RIBEIRO NETO*, Irya Fernandes Martins MAIA \& Milena CHEFER \\ Centro Universitário São Camilo, Cachoeiro do Itapemiri, Espírito Santo, Brasil. \\ *Autor para correspondência: nelsoncoimbra@ saocamilo-es.br \\ http://dx.doi.org/10.18571/acbm.095
}

\section{RESUMO}

Atualmente a obesidade é considerada um problema de saúde pública e muitos são os instrumentos para controle da mesma. Entre os mais difundidos, encontram-se as cirurgias bariátricas, dentre elas a gastrectomia vertical por videolaparoscopia, que se trata de um procedimento relativamente novo onde basicamente o estômago é transformado em um tubo, com capacidade de 80 a 100ml. O objetivo deste estudo foi analisar quantitativamente a melhora da função pulmonar e da força muscular respiratória entre o pré e pós-operatório de cirurgia bariátrica, por meio do acompanhamento em três momentos na realização de espirometria e manovacuometria (pré-operatório, pós-operatório imediato e pós-operatório tardio). A pesquisa foi realizada com 4 pacientes, submetidos à gastrectomia vertical por videolaparoscopia, na Santa Casa de Misericórdia de Cachoeiro de Itapemirim-ES, operados pelo mesmo médico, entre fevereiro e junho de 2015. Os resultados encontrados na pesquisa não demostraram diferenças estatísticas significativas entre os momentos de coleta de dados pré e pós-operatórios. Houve redução de peso e consequente redução do İndice de Massa Corporal (IMC) após o procedimento, e sugere-se que sejam realizados mais estudos com pacientes bariátricos a fim de se analisar a alteração da função pulmonar antes e após a cirurgia.

Palavras-chave: Cirurgia Bariátrica; Espirometria; Força Muscular.

\begin{abstract}
Quantitative analysis of lung function and respiratory muscle strength improvement between pre and post surgery of vertical sleeve gastrectomy by videolaparoscopic. Nowadays the obesity is considered a public health problem and there are many ways to control it. Among the most commons we found the bariatric surgeries, within the vertical sleeve gastrectomy by videolaparoscopic. This is a relative new procedure where the stomach is transformed into a 2,70 to 3,40us fl oz tube. The aim of this study was to analyze the lung function and respiratory muscle strength improvement between pre and post bariatric surgery, by following three moments of spirometry and manovacuometry tests (pre surgery, immediate post surgery and late post surgery). The study was developed with 4 vertical sleeve gastrectomy by videolaparoscopic patients, from "Santa Casa de Misericórdia" in Cachoeiro de Itapemirim-ES. All of them were operated by the same surgeon between February and June 2015. The results do not show significant statistical differences among the data collect steps on pre and post surgery. Afterwards there was weight and Body Mass Index (BMI) reduction, and we suggest more studies with bariatric patients due to analyze the lung function changes before and after the surgery.
\end{abstract}

Keywords: Bariatric Surgery; Spirometry; Muscle Strength. 


\section{Introdução}

De acordo com Soares et al (2011), a obesidade se caracteriza pelo aumento do Índice de Massa Corpórea (IMC), sobretudo pelo acúmulo de gordura generalizada ou localizada. Ela se enquadra no grupo de doenças crônico-degenerativas não transmissíveis e não infecciosas, sendo um dos mais graves problemas de saúde pública, especialmente em países em desenvolvimento, o que levou à condição de epidemia global.

A Associação Brasileira para o Estudo da Obesidade e da Síndrome Metabólica (ABESO), embasada em estudos da Organização Mundial de Saúde (OMS), aponta que, até 2025, cerca de 2,3 bilhões de adultos estejam com sobrepeso; e mais de 700 milhões, obesos. Segundo o mapa de obesidade publicado pela (ABESO), a região sudeste brasileira, apresenta $50,45 \%$ de sua população adulta com excesso de peso (ABESO, 2009).

O tratamento da obesidade é complexo e multifatorial objetivando a melhora da qualidade de vida e a diminuição da mobilidade e mortalidade de pacientes obesos por meio da redução da quantidade de massa corporal, em especial de gordura. O primeiro tratamento é o clínico, baseado em dieta, exercícios físicos e tratamento farmacológico. Nos casos em que a obesidade traz prejuízos à saúde e o tratamento clínico se mostra ineficaz, o tratamento cirúrgico é recomendado, dentro de suas indicações e contraindicações.

Marcelino e Patrício (2011) destacam que dados da Sociedade Brasileira de Cirurgia Bariátrica e Metabólica (SBCBM) apontam que em 2010 foram realizadas 64,04 mil cirurgias no país, aumento de $275 \%$ em relação a 2003; ano em que os primeiros registros foram coletados; e de $33 \%$ em relação a 2009. Especificamente pelo SUS, o número de cirurgias aumentou $23,7 \%$ entre 2007 e 2009, chegando a 3.681 intervenções, mesmo com filas de espera de até oito anos. Em hospitais vinculados ao SUS o número de cirurgias aumentou quase $800 \%$ entre 2001 e 2010. Em unidades particulares, o crescimento registrado na última década é menor, cerca de $300 \%$. Esses números fazem do Brasil o segundo colocado no ranking de cirurgias bariátricas, atrás apenas dos Estados Unidos, com 300 mil procedimentos em 2010.

Dentre as várias técnicas cirúrgicas para obesidade, Zeve et al (2012), citam a gastrectomia vertical por videolaparoscopia como um procedimento relativamente novo, sendo difundida nos anos 2000. Branco Filho et al (2011) evidencia a boa eficácia desta técnica, sobre o controle da hipertensão e de doenças dos lípides. Nesse procedimento o estômago é transformado em um tubo, com capacidade de 80 a $100 \mathrm{ml}$. Com a remoção de parte relevante da grande curvatura gástrica, cria-se um estômago tubular com pequena capacidade volumétrica, dando a característica restritiva à operação. Como é realizado por videolaparoscopia, são feitas de 4 a 5 mini-incisões de 0,5 a 1,2cm cada uma, por onde passam as cânulas e a câmera de vídeo.

Branco Filho et al (2011) em seus estudos, afirma que a gastrectomia vertical por videolaparoscopia, constitui um dos métodos cirúrgicos seguros e eficazes, apresentando baixa morbimortalidade, bons resultados pós-operatórios, baixo índice de complicações, redução do tempo de cirurgia e dos dias de internação, diminuição do risco de infecção e a possibilidade de o paciente voltar às atividades normais em menos tempo.

Como descreve Gontijo et al (2011), a obesidade pode comprometer o sistema respiratório devido ao acúmulo de gordura peritorácica e abdominal, diminuindo o volume de reserva expiratório (VRE) e a capacidade residual funcional (CRF), promovendo também alterações na relação ventilação/perfusão (V/Q) pela hipoxemia de repouso e em posição supina, provavelmente devido ao fechamento de pequenas vias aéreas. Abreu et al (2014), explica que após cirurgia abdominal, o paciente gera um padrão restritivo por medo de respirar. Esse padrão resulta em fraqueza da musculatura ventilatória e disfunção diafragmática, reduzindo as funções respiratórias, a capacidade de expectoração, culminando em complicações pulmonares. Diante disso, dois testes de função pulmonar são frequentemente utilizados: espirometria e manovacuometria. 
A espirometria, segundo Costa e Jarnarni (2001) e Gontijo et al (2011), também é conhecida como prova de função pulmonar que mede pelo ar inalado e exalado pelos pulmões, dentre outros, os seguintes volumes e capacidades pulmonares: capacidade vital forçada (CVF); volume expiratório forçado no primeiro segundo $\left(\mathrm{VEF}_{1}\right)$; fluxo expiratório forçado entre $25 \%$ e 75\% da CVF (FEF 25\%-75\%); relação $\mathrm{VEF}_{1} / \mathrm{CVF}$ e pico de fluxo expiratório (PFE). Tem por finalidade detectar a presença ou ausência de disfunção pulmonar, avaliar a evolução clínica de uma pneumopatia e parametrizar recursos terapêuticos por meio de testes pré e pós-intervenção terapêutica, avaliar o risco cirúrgico, direcionar condutas em pacientes cardiopatas e subsidiar a avaliação da saúde do trabalhador, especialmente no controle de riscos industriais. Há a necessidade de alguns cuidados para a realização da técnica, pois apesar de ser considerada uma técnica segura, pode ocorrer, eventualmente, aumento da pressão intracraniana, síncope, tontura, dor torácica, tosse paroxística, risco de infecções, broncoespasmo e pneumotórax.

Novaes et al (2013) explicam, baseados na literatura, que a força da musculatura respiratória pode ser mensurada pela medida da pressão inspiratória máxima e da pressão expiratória máxima (PImáx e PEmáx), através do manovacuômetro. A medida de PImáx é considerada como um índice da força dos músculos inspiratórios, principalmente do músculo diafragma, e a PEmáx refere-se à força dos músculos expiratórios, principalmente dos músculos abdominais.

Ainda de acordo com Novaes et al (2013), a mensuração da força dos músculos respiratórios tem uma vasta aplicação, pois possibilita o diagnóstico precoce da fraqueza em músculos respiratórios, ajudando o profissional da saúde, em especial o fisioterapeuta, a estabelecer o protocolo de treinamento físico geral e em particular da musculatura respiratória.

Com o objetivo de analisar quantitativamente a melhora da função pulmonar e da força muscular respiratória entre o pré e pós-operatório de cirurgia bariátrica, esta pesquisa vai de encontro aos estudos de Mancini (2001), que afirma que a função pulmonar deve ser acompanhada no paciente obeso durante todo seu tratamento, seja ele cirúrgico ou clínico, a fim de se quantificar os resultados previstos e os alcançados, para melhor acompanhamento.

\section{Materiais e Métodos}

Este é um estudo quantitativo, de observação estruturada não participante, de caráter exploratório e comparativo, cujo projeto de pesquisa foi submetido e aprovado pelo Comitê de Ética e Pesquisa do Centro Universitário São Camilo sob o número 922.838, em 10 de fevereiro de 2015, sendo desenvolvido com pacientes adultos obesos, que foram submetidos à cirurgia de gastrectomia vertical por videolaparoscopia. Todos os pacientes selecionados possuíam o mesmo médico cirurgião e realizaram o procedimento cirúrgico no Hospital Santa Casa de Misericórdia, em Cachoeiro de Itapemirim-ES, no período de Fevereiro a Junho de 2015.

Para composição da amostra os seguintes critérios de inclusão foram considerados: Índice de Massa Corporal igual ou superior a $35 \mathrm{~kg} / \mathrm{m}^{2}$ antes da cirurgia e/ou ter indicação para cirurgia bariátrica; idade entre 18 e 59 anos. Foram estabelecidos os seguintes critérios de exclusão para a amostra: pacientes que tenham alterações cognitivas aparentes, não conseguindo compreender e realizar os comandos para os testes; que possuam idade inferior a 18 anos e superior a 59 anos; que tenham sido hospitalizados entre a alta hospitalar pós-cirúrgica e a coleta de dados do pósoperatório tardio; e pacientes tabagistas e/ou que apresentassem sintomas de doenças respiratórias crônicas. Além disso, nenhum participante passou por atendimentos de fisioterapia respiratória, seja no pré ou no pós-operatório.

Foram selecionados 4 pacientes e todos foram instruídos sobre a pesquisa: os objetivos, seus riscos e benefícios, e quanto à opção em não participar ou desistir a qualquer fase da coleta de dados. Para isso, firmaram um termo de consentimento livre e esclarecido como voluntários. 
Durante o período de coleta de dados, nenhum participante se recusou a fazer parte do grupo amostral ou sequer desistiu após o consentimento formalizado.

A coleta de dados foi realizada em três etapas, sendo uma no pré-operatório (dia de internação cirúrgica), a segunda no pós-operatório imediato (1 dia de pós-operatório, em ambiente hospitalar) e outra no pós-operatório tardio, de 15 a 52 dias após o procedimento, pois dependia-se do retorno clínico dos pacientes ao seu médico cirurgião. Em cada fase foram realizados testes de força muscular respiratória através da manovacuometria, avaliando os índices de $\mathrm{P}_{\mathrm{i}} \mathrm{MAX}$ e $\mathrm{P}_{\mathrm{e}} \mathrm{MAX}$ (Pressão Inspiratória Máxima e Pressão Expiratória Máxima), além de exame de espirometria, analisando a CVF (Capacidade Vital Forçada), VEF $_{1}$ (Volume Expirado no primeiro segundo) e PFE (Pico de Fluxo Expiratório). Para as mensurações foram utilizados um manovacuômetro com limite operacional de $-300 \mathrm{a}+300 \mathrm{~cm}_{2} \mathrm{O}$ e um espirômetro portátil da marca MIR (Medical International Research), modelo Spirotel ${ }^{\circledR}$. Foram aferidas 3 medidas válidas para cada teste, sendo adotadas as de melhor valor, de acordo com as diretrizes da American Thoracic Sociey (ATS).

Para a coleta no manovacuômetro, e espirômetro, os indivíduos foram posicionados sentados e em repouso mínimo de 10 minutos, utilizando um clipe nasal durante a execução. Para a PiMAX os participantes foram instruídos a expirar até o volume residual e então realizar a inspiração forçada máxima. Para coleta de PeMAX o indivíduo era instruído a inspirar até o final do volume de reserva inspiratório e então realizar a expiração forçada. Para a coleta dos valores espirométricos, $\left(\mathrm{CVF}, \mathrm{VEF}_{1}\right.$ e PFE) o paciente era orientado a realizar uma inspiração até o final do volume de reserva inspiratório e em seguida abraçar o bocal com os lábios e realizar uma expiração forçada de glote aberta, rápida, e contínua até o volume residual. Todos os testes foram acompanhados por fisioterapeuta especialista em pneumologia para validação dos resultados.

Os dados tabulados foram analisados primeiramente pelo teste de normalidade de Shapiro Wilk, e posteriormente pelo teste não paramétrico de Kruskal Wallis, seguido do teste pós-hoc de Dunn, adotando-se o valor de significância de $\mathrm{p}<0,05$. Também foi utilizado o teste de Wilcoxon para comparar especificamente a aferição pré-operatória com a pós-operatória, seja imediata, ou tardia, também considerando $\mathrm{P}<0,05$. Para a análise dos dados foi utilizado o programa estatístico GraphPad Prism $5^{\circledR}$.

\section{Resultados}

Participaram do estudo 4 pacientes com idade média de 37,25 anos, nos quais $75 \%$ eram do gênero feminino e $25 \%$ do masculino. A média de IMC no pré-operatório foi de $46,59 \mathrm{~kg} / \mathrm{m}^{2}$, apresentando 3 pacientes com IMC entre 37,76 e $45,9 \mathrm{~kg} / \mathrm{m}^{2}$ e um paciente com IMC $>60 \mathrm{~kg} / \mathrm{m}^{2}$. Considerando o pós-operatório tardio, todos os pacientes obtiveram perda de peso, numa média de $12,075 \mathrm{~kg}$, com média de IMC de $42,19 \mathrm{~kg} / \mathrm{m}^{2}$, onde 2 pacientes apresentaram IMC entre 32,37 e $38,05 \mathrm{~kg} / \mathrm{m}^{2}$ e 2 pacientes ainda apresentavam IMC acima de $40 \mathrm{~kg} / \mathrm{m}^{2}\left(41,86\right.$ e $\left.56,50 \mathrm{~kg} / \mathrm{m}^{2}\right)$. A tabela 1 demonstra os dados antropométricos de caracterização dos sujeitos da pesquisa. 
Tabela 1: Caracterização antropométrica da amostra.

\begin{tabular}{ccccc}
\hline \multirow{2}{*}{ AMOSTRA } & \multirow{2}{*}{ GÊNERO } & \multirow{2}{*}{ IDADE (anos) } & \multicolumn{2}{c}{ IMC $\left(\mathbf{k g} / \mathbf{m}^{\mathbf{2}}\right)$} \\
& & & Pré-OP & Pós-OP T \\
\hline N1 & Feminino & 36 & 37,76 & 32,37 \\
N2 & Feminino & 46 & 45,9 & 41,86 \\
N3 & Masculino & 38 & 62,03 & 56,50 \\
N4 & Feminino & 29 & 40,67 & 38,05 \\
\hline
\end{tabular}

Pré-OP - Pré-operatório. Pós-OP T - Pós-operatório tardio.

$\mathrm{Na}$ coleta de dados do pré-operatório, todos os pacientes apresentaram na análise da espirometria função pulmonar normal, assim como no pós-operatório tardio. No pós-imediato apresentaram restrição pulmonar, sendo 3 deles restrição moderada e 1 restrição moderada grave, entretanto, a diferença não foi estatisticamente significativa quando comparados o pré-operatório com o pós-operatório imediato ou com o pós-operatório tardio.

No gráfico 1 observamos os valores de PiMAX no pré-operatório $(-92,5 \pm 35,94)$, pósoperatório imediato $(-51,25 \pm 13,15)$ e pós-operatório tardio $(-90 \pm 27,08)$, tendo sido adotado o teste não paramétrico de Kruskal-Wallis ( $\mathrm{p}=0,0448)$, considerando, portanto, diferença entre as médias aferidas.

Porém, ao se comparar o pré-operatório com o pós-operatório imediato ou com o pósoperatório tardio, por meio do teste não paramétrico de Wilcoxon, não se constatou diferença estatística entre as medidas pareadas, onde obteve-se um valor de $\mathrm{p}=0,0625$ para a primeira comparação e de $\mathrm{p}=0,500$ para a segunda.

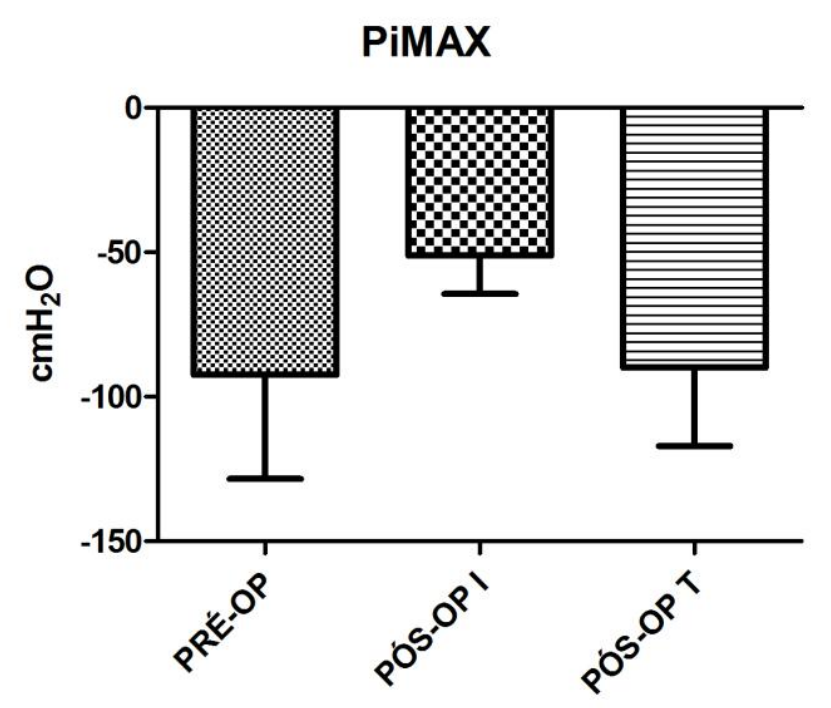

Gráfico 1: valores de PiMAX aferidos no pré, no pós imediato e no pósoperatório tardio, submetidos ao teste de Kruskal-Wallis para análise de variância $(p=0,0448)$.

No gráfico 2 observamos os valores de PeMAX no pré-operatório $(94,25 \pm 49,72)$, pósoperatório imediato $(51,25 \pm 23,23)$ e pós-operatório tardio $(117,5 \pm 35)$, Observando-se valor de $\mathrm{p}=0,0370$ para o teste de Kruskal-Wallis.

Porém, ao se comparar o pré-operatório com o pós-operatório imediato ou com o pósoperatório tardio, obteve-se valor de $\mathrm{p}=0,0625$ para ambas as comparações, por meio do teste de Wilcoxon. 


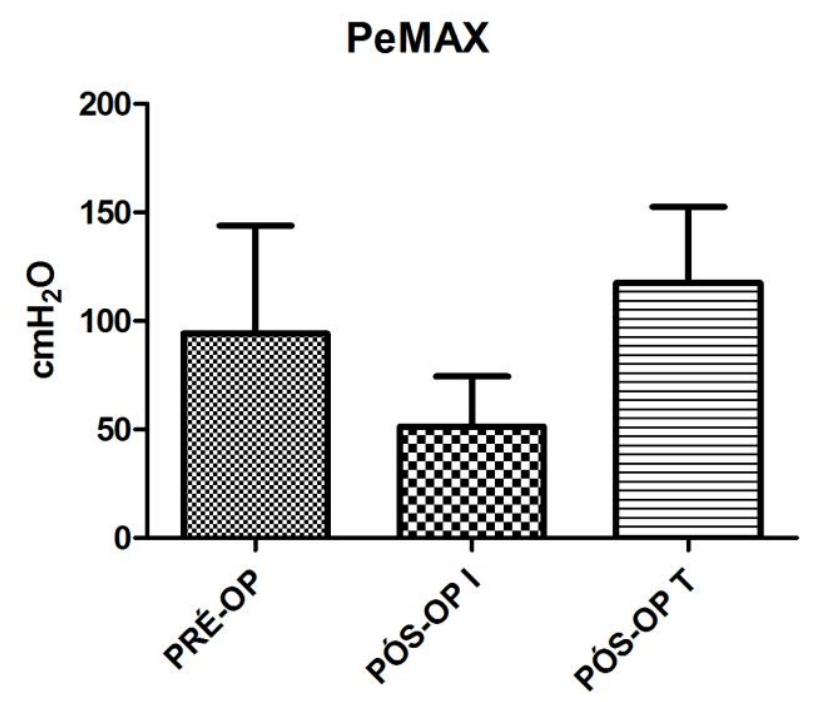

Gráfico 2: valores de PeMAX aferidos no pré, no pós imediato e no pósoperatório tardio, submetidos ao teste de Kruskal-Wallis para análise de variância $(p=0,0370)$.

Em nossos achados espirométricos, quando analisamos as variáveis de CVF, PFE e $\mathrm{VEF}_{1}$ no pré $(3,74 \pm 1,103 ; 6,145 \pm 1,908 ; 3,835 \pm 1,599)$, no pós imediato $(2,418 \pm 0,6676 ; 4,710 \pm$ $2,503 ; 1,965 \pm 0,6653)$ e no pós-operatório tardio $(3,768 \pm 1,069 ; 7,073 \pm 2,109 ; 3,130 \pm 1,004)$, identificamos que apenas a capacidade vital forçada apresentou diferença entre as três médias para o teste de Kruskal-Wallis, com valor de $\mathrm{p}=0,0476$ (gráficos 3, 4 e 5). Já no pareamento pelo teste de Wilcoxon não encontramos diferenças estatísticas entre as aferições do pré-operatório e pós-operatório imediato, ou do pré com o pós-operatório tardio.

\section{CVF}

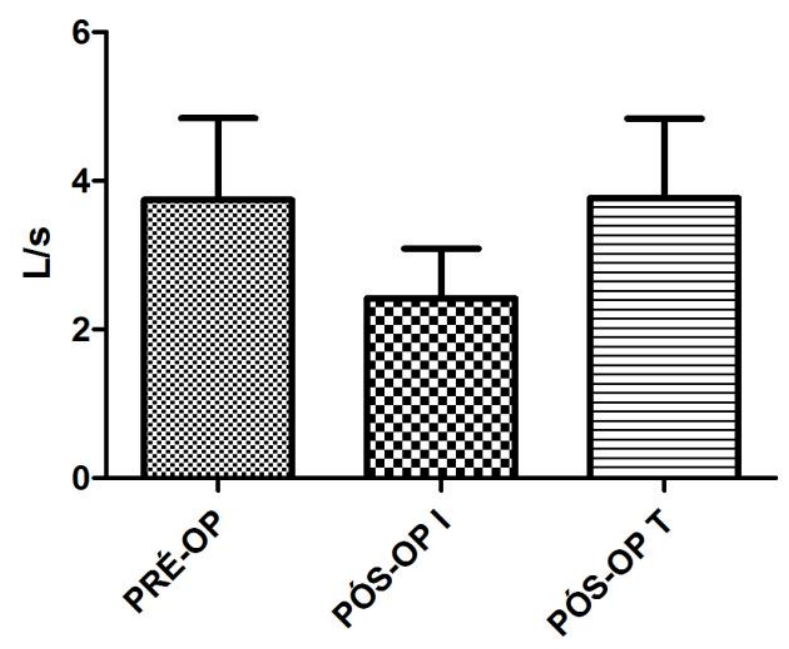

Gráfico 3: valores de CVF aferidos no pré, no pós imediato e no pósoperatório tardio, submetidos ao teste de Kruskal-Wallis para análise de variância $(\mathrm{p}=0,0476)$, e teste de Wilcoxon aplicado na comparação entre Pré-

OP X Pós I (p=0,0625); Pré-OP X Pós T (p=0,4375). 


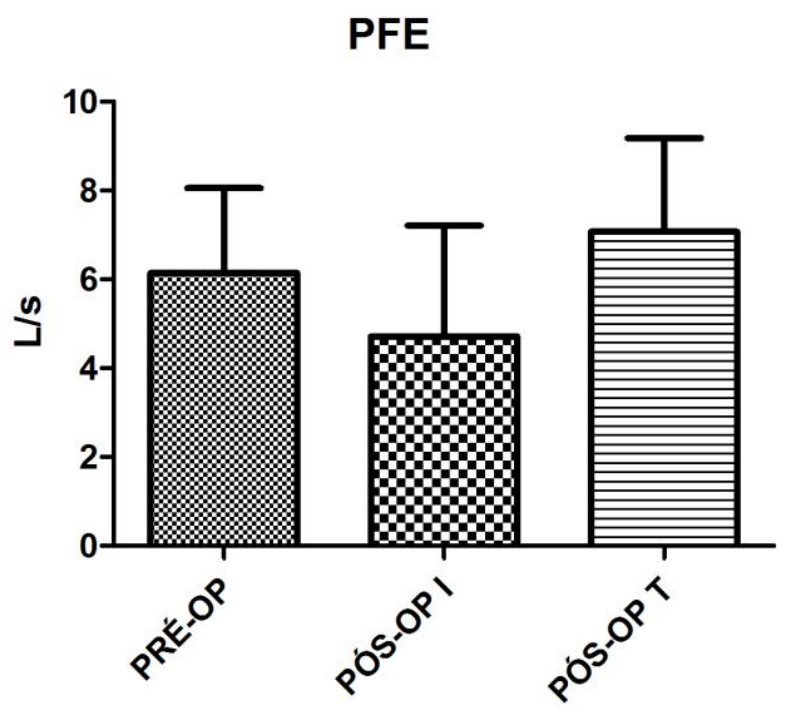

Gráfico 4: valores de PFE aferidos no pré, no pós imediato e no pósoperatório tardio, submetidos ao teste de Kruskal-Wallis para análise de variância $(p=0,2457)$, e teste de Wilcoxon aplicado na comparação entre PréOP X Pós I (p=0,0625); Pré-OP X Pós T (p=0,0625).

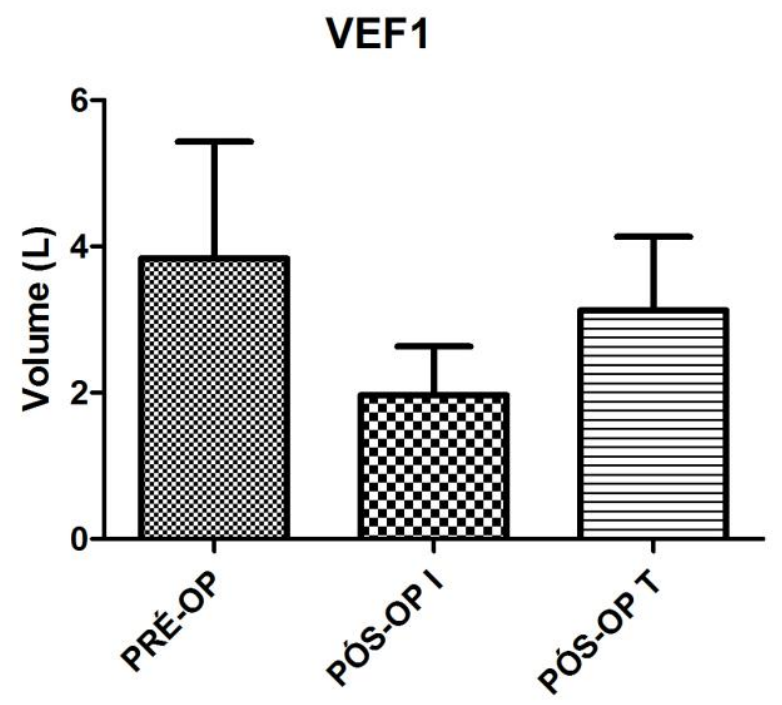

Gráfico 5: valores de $\mathrm{VEF}_{1}$ aferidos no pré, no pós imediato e no pósoperatório tardio, submetidos ao teste de Kruskal-Wallis para análise de variância $(p=0,0837)$, e teste de Wilcoxon aplicado na comparação entre PréOP X Pós I (p=0,0625); Pré-OP X Pós T ( $\mathrm{p}=0,3125)$.

Possivelmente em uma amostra maior e adotando-se separação de grupos por gênero, poderíamos obter diferenças estatisticamente significativas entre as variáveis aferidas. 


\section{Discussão}

A Associação Brasileira para o Estudo da Obesidade e da Síndrome Metabólica (ABESO), embasada em estudos da Organização Mundial de Saúde (OMS), descreve que a prevalência mundial de obesidade mais do que dobrou nas últimas três décadas. Em 2008, $10 \%$ dos homens e $14 \%$ das mulheres do mundo eram obesos. Em 2008-2009, um estudo nacional brasileiro denominado Pesquisa de Orçamento Familiar (POF) diagnosticou o excesso de peso em cerca de metade dos homens e das mulheres brasileiros, também observando um aumento contínuo de excesso de peso e obesidade na população de 20 anos ou mais de 1974 até 2009 (ABESO, 2009).

Delgado e Lunardi (2011) fizeram um levantamento bibliográfico das principais complicações pós-operatórias na cirurgia bariátrica e constataram que a principal e mais frequente alteração respiratória foi a espirométrica, o que deixa os pacientes mais susceptíveis a desenvolverem complicações pulmonares propriamente ditas. A principal redução relatada é a do volume corrente, relacionada a fatores como distúrbios do sono, altos valores de IMC e distribuição corporal do tipo ginóide. Já Ebeo et al. (2002) comparou a função pulmonar nos períodos pré e pós-operatório e observou redução de até $55 \%$ da capacidade vital forçada e do volume expiratório forçado.

Scipioni et al (2011), em seu estudo com pacientes também de cirurgia bariátrica, observou melhora da função pulmonar na espirometria do pós-operatório (90 dias) apenas dos pacientes que tiveram, no pré-operatório, função pulmonar com distúrbio inespecífico. Os percentuais dos que possuíam distúrbios ventilatórios restritivo e obstrutivo, já na avaliação pré-operatória, foram mantidos, não verificando assim, diferenças significativas entre as proporções das avaliações pré e pós-operatória.

Outro estudo que evidencia a não mudança considerável de valores de espirometria, menciona que após a cirurgia e perda de peso nem todos os doentes ficaram com provas normais. “Apesar da resolução do padrão restritivo em todos eles, manteve-se a diminuição da CRF em 13 doentes, o que corresponde aos doentes com maior IMC inicial e com menor diferença na perda de peso" (GUIMARÃES et al, 2012).

Paisani et al (2005), ao analisarem a força muscular respiratória em estudo com 21 pacientes de gastroplastia, verificaram em relação ao valor do pré-operatório, queda da PiMAX de $51 \%, 26 \%$ e $14 \%$ no primeiro, terceiro e quinto dias de pós-operatório ( $\mathrm{p}<0,001, \mathrm{p}<0,001$, $\mathrm{p}=0,028$ ). A PeMAX média apresentou queda, também em relação ao valor do pré-operatório, de $39 \%, 26 \%$ e $15 \%$ no primeiro, terceiro e quinto dias de pós-operatório ( $p<0,001, \mathrm{p}<0,001$ e $\mathrm{p}=0,072$ ). Desta maneira, observou-se que os valores médios de PiMAX e PeMAX cresceram de maneira linear nos momentos de pós- operatório ( $\mathrm{p}=0,684, \mathrm{p}=0,071)$.

Aguiar et al (2009), em discussão no seu estudo, cita que resultado semelhante já foi encontrado com um grupo de 19 pacientes (12 mulheres e 7 homens) submetidos à cirurgia bariátrica, sendo avaliados no pré-operatório, $2^{\circ}$ dia de pós-operatório (PO), $10^{\circ} \mathrm{PO}$ e $30^{\circ} \mathrm{PO}$. Como resultado foi percebido a recuperação dos valores pré- operatórios de PiMAX entre o $2^{\circ}$ $\mathrm{PO}$ e o $10^{\circ} \mathrm{PO}$, não tendo recuperado os valores de PeMAX até o $30^{\circ} \mathrm{PO}$.

Nassif et al (2004) fez uma avaliação utilizando o manovacuômetro no pré-operatório e terceiro dia pós-operatório, com 23 pacientes indicados a cirurgia bariátrica, porém não aplicou nenhum protocolo de tratamento, e contraditoriamente aos resultdos dos autores Paisani et al (2005), concluiu que os pacientes submetidos à cirurgia bariátrica apresentam diminuição significativa apenas dos valores de PeMAX no pós-operatório.

Canavezi et al (2012), ao analisarem as variáveis CVF, VEF1 e VEF1/CVF e compararem os valores obtidos entre o pré-operatório e o pós-operatório, constataram que os resultados encontrados para o Índice de Tiffeneau caracterizavam redução da função pulmonar no pósoperatório, com melhora após sétimo dia de cirurgia, mas mantendo valores referenciados como normais, como já descrito na literatura. Esta variação é uma normalidade porque ocorrem 
alterações funcionais nos músculos respiratórios, principalmente no diafragma. Ocorre uma irritação do centro tendíneo pelo $\mathrm{CO}_{2}$ que provoca disfunção mecânica e gera reflexos inibitórios, com redução da descarga do nervo frênico e consequentemente redução da motilidade diafragmática.

\section{Conclusão}

Nos últimos anos a cirurgia bariátrica se revelou um importante instrumento para controle da obesidade. Assim, diante do desenvolvimento de fatores que acometem a funcionalidade do sistema respiratório no paciente obeso, e candidato à realização da cirurgia, é de extrema importância a avaliação e comparação da função pulmonar dessa população.

A redução de peso e consequente redução do IMC se mostrou semelhante a de outros estudos referenciados, e foi possível identificar diferenças entre as medidas aferidas nas três etapas.

Porém, possivelmente devido à pequena amostra disponibilizada para a pesquisa, em nossos achados verificamos que não houve alteração significativa dos valores de espirometria e manovacuometria entre as fases de pré-operatório e pós-operatório imediato ou de pré e pósoperatório tardio.

Sugerimos que sejam realizados mais estudos com pacientes bariátricos a fim de se analisar a alteração da função pulmonar antes e após a cirurgia, e que sejam observadas características entre gêneros, o que não foi possível nesta pesquisa. Também aferimos que a literatura ainda carece de estudos comparativos entre métodos cirúrgicos distintos.

\section{Referências}

ABREU, Ana Paula Monteiro et al. Função pulmonar e força muscular respiratória em pacientes submetidas à cirurgia oncológica de mama. Revista Brasileira de Cancerologia, Rio de Janeiro, n.60, v.2, p.151-57, abr./jun. 2014.

ABESO. Associação Brasileira para o Estudo da Obesidade e da Síndrome Metabólica. Diretrizes Brasileiras de Obesidade. 3 ed. São Paulo: AC Farmacêutica, 2009.

AGUIAR, A. C. et al. Análise da atuação fisioterapêutica em relação à força muscular respiratória em pacientes submetidos à cirurgia bariátrica. Revista Movimenta, Goiânia, v.2, n.2, p.54-8, 2009.

BRANCO FILHO, Alcides José et al. Tratamento da obesidade mórbida com gastrectomia vertical. Arquivo Brasileiro de Cirurgia Digestiva-ADCD, Curitiba, v.24, n.1, p.52-4, jan./mar. 2011.

CANAVEZI, Alessandro; RIVA, Mirele; CAIERÃO, Quélen Milani. Avaliação espirométrica de pacientes obesos submetidos a tratamento cirúrgico para a obesidade mórbida. EFDeportes.com, v.17, n.170, jul. 2012. Disponível em: < http://www.efdeportes.com/efd170/avaliacaoespirometrica-de-pacientes-obesos.htm>. Acessado em: 12 de junho de 2015.

COSTA, D.; JARNARNI, M. Bases fundamentais da espirometria. Revista brasileira de Fisioterapia, São Carlos-SP, v.5, n.2, p.95-102, abr. 2001. 
DELGADO, Priscila Martins; LUNARDI, Adriana Cláudia. Complicações respiratórias pósoperatórias em cirurgia bariátrica: revisão da literatura. Fisioterapia e Pesquisa, São Paulo, v.18, n.4, p. 388-92, out./dez. 2011.

GUIMARÃES, C.; MARTINS, M. V.; SANTOS, J. Moutinho dos. Função pulmonar em doentes obesos submetidos à cirurgia bariátrica. Revista Portuguesa de Pneumologia, Coimbra, v.18, n.3, p.115-19, mai./jun. 2012.

GONTIJO, Patrícia Lúcia et al. Correlação da espirometria com o teste de caminhada de seis minutos em eutróficos e obesos. Revista da Associação Médica Brasileira, São Paulo, v.4, n.57, p.387-93, abr. 2011.

MARCELINO, Liete Francisco; PATRÍCIO, Zuleica Maria. A complexidade da obesidade e o processo de viver após a cirurgia bariátrica: uma questão de saúde coletiva. Ciência e Saúde Coletiva, Rio de Janeiro, v.16, n.12, p.4767-76, mar. 2011.

MANCINI, Marcio C. Obstáculos diagnósticos e desafios terapêuticos no paciente obeso. Revista Brasileira de Endocrinologia e Metabologia, São Paulo, v.45, n.6, p.584-90, dez. 2001.

NASSIF, P. A. et al. Avaliação da força e endurance da musculatura respiratória em pacientes submetidos à gastroplastia. Revista Médica do Paraná, Curitiba, v.62, n.2, p.10-4, jul./dez. 2004.

NOVAES, Priscila Alves; SANCHEZ, Eliane Gouveia de Morais; SANCHEZ, Hugo Machado. Medida das pressões respiratórias máximas em jovens saudáveis antes e após manobra de liberação diafragmática. Movimento \& saúde, Revista Inspirar, v.5, n.2, p.1-5, jun./jul. 2013.

PAISANI, Denise de Moraes; CHIAVEGATO, Luciana dias; FARESIN, Sonia Maria. Volumes, capacidades pulmonares e força muscular respiratória no pós-operatório de gastroplastia. Jornal Brasileiro de Pneumologia, Brasília, v.31, n.2, p.125-32, mar./abr. 2005.

SCIPIONI, G. et al. Função pulmonar de obesos mórbitos submetidos à cirurgia bariátrica. Fisioterapia em Movimento, Curitiba, v.24, n.4, p.621-27, out./dez. 2011.

SOARES, K. K. D. et al. Avaliação do desempenho físico e funcional respiratório em obesos. Fisioterapia em Movimento, Curitiba, v.24, n.4, p.697-704, out./dez. 2011.

ZEVE, Jorge Luiz de Mattos; NOVAIS, Poliana Oliveira; OLIVEIRA JÚNIOR, Nilvan de. Técnicas em cirurgia bariátrica: uma revisão da literatura. Revista Ciência \& Saúde, Porto Alegre, v.5, n.2, p.132-40, jul./dez. 2012. 